\title{
Finite element analysis of true and pseudo surface acoustic waves in one-dimensional phononic crystals
}

\author{
B. Graczykowski, ${ }^{1, a)}$ F. Alzina, ${ }^{1}$ J. Gomis-Bresco, ${ }^{1}$ and C. M. Sotomayor Torres ${ }^{1,2}$ \\ ${ }^{1}$ Catalan Institute of Nanoscience and Nanotechnology (ICN2), CSIC and the Barcelona Institute of Science \\ and Technology, Campus UAB, Bellaterra, 08193 Barcelona, Spain \\ ${ }^{2}$ ICREA_Institucio Catalana de Recerca i Estudis Avançats, 08010 Barcelona, Spain
}

(Received 2 October 2015; accepted 31 December 2015; published online 14 January 2016)

\begin{abstract}
In this paper, we report a theoretical investigation of surface acoustic waves propagating in onedimensional phononic crystal. Using finite element method eigenfrequency and frequency response studies, we develop two model geometries suitable to distinguish true and pseudo (or leaky) surface acoustic waves and determine their propagation through finite size phononic crystals, respectively. The novelty of the first model comes from the application of a surface-like criterion and, additionally, functional damping domain. Exemplary calculated band diagrams show sorted branches of true and pseudo surface acoustic waves and their quantified surface confinement. The second model gives a complementary study of transmission, reflection, and surface-to-bulk losses of Rayleigh surface waves in the case of a phononic crystal with a finite number of periods. Here, we demonstrate that a non-zero transmission within non-radiative band gaps can be carried via leaky modes originating from the coupling of local resonances with propagating waves in the substrate. Finally, we show that the transmission, reflection, and surface-to-bulk losses can be effectively optimised by tuning the geometrical properties of a stripe. (C) 2016 AIP Publishing LLC.
\end{abstract}

[http://dx.doi.org/10.1063/1.4939825]

\section{INTRODUCTION}

Since the first study by John Strutt (3rd Lord Rayleigh) in 1885, Rayleigh surface waves (RSWs), the simplest of surface acoustic waves (SAWs), have become the subject of a large number of papers, monographs, applications, and patents. ${ }^{1-8}$ The experimental and theoretical studies of the propagation of SAWs in periodically corrugated surfaces of the 1970s and 1980s initiated informally the field of phononic crystals (PnCs). ${ }^{9-12}$ The artificial second-order periodicity, introduced by an array of grooves or metallic stripes, was shown to result in the appearance of surface Brillouin zones, zone folding, pseudo-SAWs, and in particular cases frequency band gaps. ${ }^{12-20}$ The research from the last twenty years has pointed out numerous attractive properties of PnCs over wide length and frequency scales, which can be applied to, e.g., seismic waves filters, coherent phonon sources, tunable acoustic filters, waveguides, and thermal management. $^{21-24}$

RSWs can be described as non-dispersive SAWs propagating near the free surface of a homogenous solid state medium with a finite penetration depth into bulk. Typically, in isotropic materials, the amplitude of RSWs decays exponentially to zero in a depth of several wavelengths. The propagation of RSWs in elastically anisotropic materials, such as crystals, is more complex; thus, velocity and penetration depth strongly depend on the direction, plane of propagation, and elastic anisotropy ratio. ${ }^{5,25}$ Therefore, the full analysis of RSWs requires numerical methods such as those based on the partial waves approach or elastodynamic Green

\footnotetext{
a) Author to whom correspondence should be addressed. Electronic mail: bartlomiej.graczykowski@icn.cat
}

functions. ${ }^{5-7,26,27}$ Moreover, these techniques allow investigating pseudo-RSWs, also called leaky RSWs. These waves appear in the radiative zone (or supersonic domain) of the dispersion relation diagram, defined by the sound line of the slow transverse bulk acoustic wave (T2BAW). Generally, they differ from true-RSWs in a small radiating component so that the amplitude of pseudo-RSWs does not decay completely to zero with depth. Under certain conditions, pseudoRSWs can be measured by means of ultrasonic techniques or Brillouin light scattering (BLS). ${ }^{5,25,28}$ These waves, in a broader context of true- and pseudo-SAWs propagating in PnCs, also piezoelectric, were investigated experimentally by transmission measurements ${ }^{29}$ and theoretically mostly by means of plane wave expansion (PWE) based methods. ${ }^{16,17,30,31}$ Nevertheless, in the case of theoretical studies based on the finite element method (FEM), pseudo-SAWs have been, up to now, mostly neglected by applying the cone of sound criterion. ${ }^{29,32-34}$ Although this approach enables sorting surface-like solutions and overcomes the limitations of FEM, it does not appear to be well-founded. For example, it fails in the case of true-SAWs that may appear at some isolated points in the radiative zone. ${ }^{18,35}$

In the present work, we consider theoretically SAWs of sagittal polarisation in finite size 1D PnCs (see Fig. 1) propagating normally to the stripes. Contrary to the previous reports, we show a way to sort surface-like solutions and distinguish true- and pseudo-SAWs within a framework of the FEM eigenfrequency analysis. With this background, we comparatively investigate finite $\mathrm{PnCs}$ for which transmission, reflection, and surface-to-bulk losses are calculated by the frequency response FEM model. Here, we propose a new procedure, which basically assumes the excitation of RSWs 
(a)

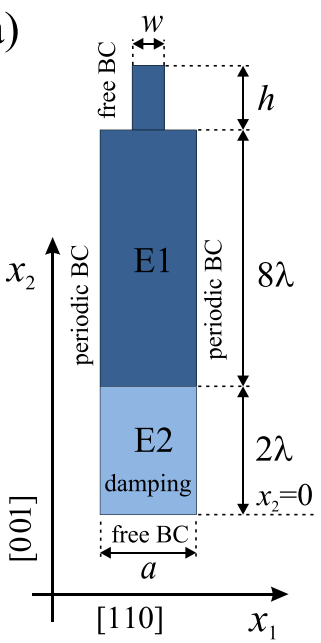

(b)

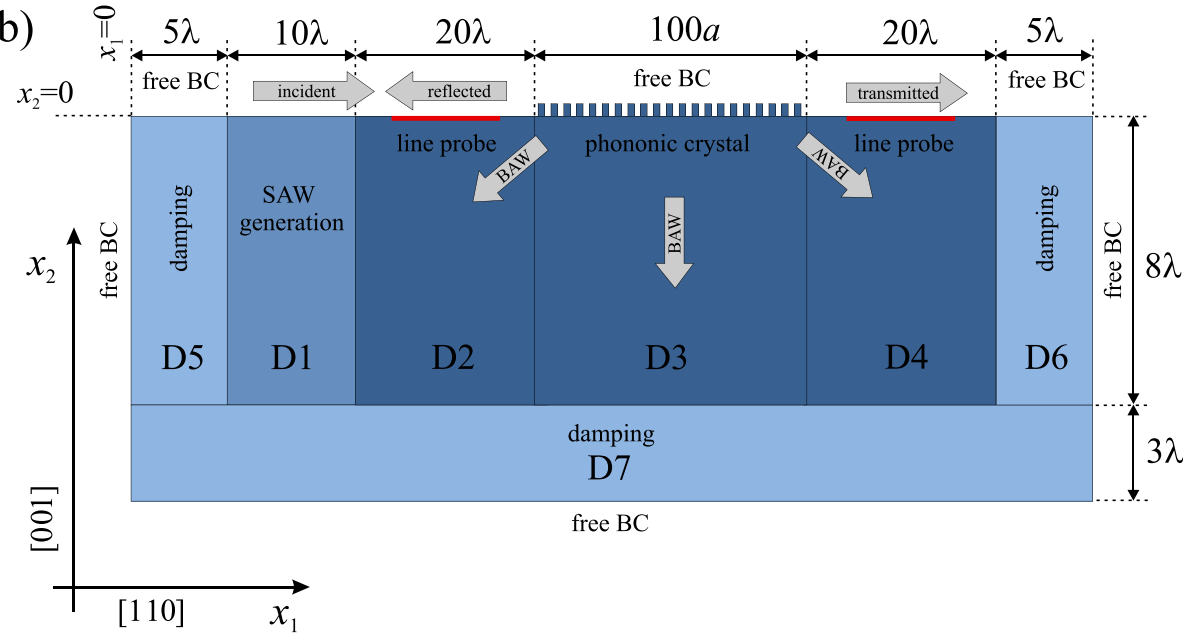

FIG. 1. Illustration of FEM models used in (a) eigenfrequency and (b) frequency response studies (description in the text).

without bulk-like components and a Fourier transform-based analysis of transmitted and reflected RSWs. Besides the novelty of the provided models, which can be extended to $3 \mathrm{D}$, our findings show the importance of pseudo-SAWs in the design and optimisation of PnCs based high efficiency acoustic filters, reflectors, or waveguides.

\section{FEM MODEL}

\section{A. Eigenfrequency study}

Rayleigh surface waves propagating in anisotropic media can be described as a specific superposition of three bulk acoustic waves (BAWs), which satisfies the stress free boundary condition. ${ }^{5,7,26}$ Therefore, the general and appropriate FEM analysis requires a 3D study. Fortunately, for isotropic materials or high symmetry planes and directions of crystals, where the displacement component perpendicular to the sagittal plane equals zero, this problem can be simplified to a $2 \mathrm{D}$ space. The presented model considers SAWs propagating in the [110] direction of the (001) plane of Si, where the above condition is satisfied. In this case, SAWs exhibit a unique feature resulting from the elastic anisotropy. Namely, the subsonic RSW degenerates with a T2BAW while the true-RSW appears in the pseudo-RSW branch lying in the supersonic domain. ${ }^{5,7}$ This particular case, where the phase velocity of RSWs is higher than that of the slowest bulk wave, has its consequences for FEM calculations of SAWs in homogenous anisotropic materials and surface PnCs as well. All FEM-based studies of SAWs in the literature use the approach, which allows overcoming the limitation in a form of a finite unit cell. Simply, the elastic halfspace is imitated by a thick slab with fixed and free boundary conditions for the bottom and upper planes, respectively, ensuring an exponential decay of the SAWs amplitude with depth. The thickness of this slab is usually arbitrarily defined and, according to the commonly accepted penetration depth of SAWs, set to be of several SAW wavelengths. ${ }^{25,29,32,36,37}$ However, a more rigorous definition should include the elastic anisotropy ratio, direction, and plane of propagation and possible existence of pseudo-SAWs.
In this paper, we focus on specific 1D surface PnCs made of rectangular stripes on the (001) plane of silicon and periodic in the [110] direction. The dispersion relation of sagittal SAWs propagating normally to the stripes was determined by looking for mechanical eigenmodes of the unit cell, depicted in Fig. 1(a). The corresponding eigenfrequencies $f=\omega / 2 \pi$ were calculated by solving the elastodynamic equation of motion

$$
-\rho \omega^{2} u_{i}-\frac{\partial \sigma_{i j}}{\partial x_{j}}=0,
$$

where the summation convention $(i=\{1,2,3\})$ is applied, $\rho$ describes the mass density, and $u_{i}$ are components of the displacement. The Cauchy stress tensor, given by $\sigma_{i j}$, is defined by the Hooke's law

$$
\sigma_{i j}=C_{i j k l} u_{k l}
$$

Here, $u_{i j}$ is the infinitesimal strain tensor expressed as

$$
u_{i j}=\frac{1}{2}\left(\frac{\partial u_{i}}{\partial x_{j}}+\frac{\partial u_{j}}{\partial x_{i}}\right)
$$

$C_{i j k l}$ is the fourth rank elastic tensor, which in the Voigt notation is given by the $6 \times 6$ symmetric elastic matrix $C_{K L}$. For cubic silicon, $C_{K L}$ has three non-zero and independent components: $C_{11}, C_{12}$, and $C_{44}$. The specific crystallographic orientation of the system, where $x_{1} \|[110]$ and $x_{2} \|[001]$, imposes a transformation of $C_{i j k l}$ according to the formula $C_{\text {pqrs }}^{\prime}=a_{p i} a_{q j} a_{r k} a_{s l} C_{i j k l}$, where $a_{i j}$ are direction cosines. ${ }^{38}$ The new elastic constants in the Voigt notation are given by $C_{K L}^{\prime}$ and gathered in Table I.

TABLE I. Elastic constants of $\mathrm{Si}$ in original $C_{K L}(\mathrm{GPa})$ and rotated $C_{K L}^{\prime}$ $(\mathrm{GPa})$ coordinates. $C_{K L}^{\prime}$ that does not appear remains unchanged.

\begin{tabular}{lllllll}
\hline \hline$C_{11}$ & $C_{12}$ & $C_{44}$ & $C_{11}^{\prime}$ & $C_{33}^{\prime}$ & $C_{55}^{\prime}$ & $C_{13}^{\prime}$ \\
\hline 165.7 & 63.9 & 79.9 & 194.7 & 194.7 & 50.9 & 34.9 \\
\hline \hline
\end{tabular}


The width of the unit cell denoted as $a$ defines the lattice constant and the reciprocal lattice wave number as $G$ $=2 n \pi / a$ ( $n$ is an integer). The height $h$ and width $w$ of a particular stripe are parametric and chosen to be comparable with the lattice period. The Bloch-Floquet periodic boundary conditions (BCs) are applied for edges normal to the $x_{1}$ axis, except those of the stripe. The proposed 2D model assumes a plane strain with a zero displacement in the $x_{3}$ direction $\left(u_{3}=0\right)$. Therefore, two remaining components of the displacement $u_{1}$ and $u_{2}$ at these edges are defined to be $u_{j} \exp \left(\mathrm{i} q x_{1}\right)$, where $j=\{1,2\}$ and $q \in\langle 0, \pi / a\rangle$ is a wave number from the reduced first Brillouin zone (IBZ). The total height $H$ of the unit cell is set as wavelength dependent, i.e., $H=10 \lambda+h$, where $\lambda=2 \pi / q$. Contrary to the previous reports, we propose the unit cell to be made out of two functional domains E1 and E2 (see Fig. 1(a)) with and without damping properties, respectively. The height of the domain E1 is taken as $8 \lambda$, which is sufficient for the SAWs propagating in the chosen orientation of silicon. Nevertheless, for any other material and crystallographic orientation, this value has to be determined individually. The upper boundaries of E1, defining the stripe, are taken to be stress free. The material properties for this domain are defined by the rotated elastic constants $C_{K L}^{\prime}$ gathered in Table I and mass density $\rho=2331 \mathrm{~kg} / \mathrm{m}^{3}$.

The additional domain E2, with the height of $2 \lambda$, introduces damping into the FEM model by an artificial viscosity. ${ }^{39}$ Herein, the elastic matrix is complex and given by

$$
C_{K L}^{\prime}(E 2)=C_{K L}^{\prime}(E 1)\left[1+5 \mathrm{i}\left(\frac{2 \lambda-x_{2}}{2 \lambda}\right)^{3}\right] .
$$

As follows from the above equation, the real part of the elastic matrix of E2 is the same as for E1 while the imaginary part is position dependent. The magnitude of the viscosity is modulated by a cubic function of the position in the $x_{2}$ axis (see Fig. 1(a)). In this way, the displacement is continuously attenuated from an arbitrary value at the boundary between E1 and E2 to zero at the bottom of the unit cell. This additional damping domain introduces some general advantages to the FEM analysis of SAWs and surface PnCs. First of all, there is no need to fix the displacement on the bottom boundary, what is crucial for the study of pseudoSAWs. In principle, we avoid imposing zero displacement of the radiating term of pseudo-SAWs at a given depth, which may result in some artificial features in the dispersion of these waves. The second and the most important benefit, discussed later, makes FEM analysis capable to distinguish true- and pseudo-SAWs.

\section{B. Frequency response study}

Figure 1(b) illustrates the geometry of the FEM model described in this section, which is based on the system previously presented in Ref. 40 . However, here we use the frequency response instead of a transient study that results in a higher calculations efficiency. The structure is divided into seven functional domains (D1, D2, ..., D7) with wavelength and thereby frequency dependent sizes. The total height of the structure set to $11 \lambda$ is sufficient to ensure no influence of the boundaries on the results. By applying the equation of motion

$$
-\rho \omega^{2} u_{i}-\frac{\partial \sigma_{i j}}{\partial x_{j}}=F_{i} \exp (\mathrm{i} \phi),
$$

we solve the steady state response from harmonic loads with the amplitude of force per unit volume $F_{i}$, driving frequency $f=\omega / 2 \pi$, and phase shift $\phi$. The domain D1 acts as a generator of RSWs of given parametric frequency and arbitrary amplitude. The common and easiest approach of introducing RSWs into the system uses a point load acting in the out-ofplane direction (here $x_{2}$ ). Nevertheless, this results in evanescent bulk-like excitations, which, if the source and $\mathrm{PnC}$ are close enough, can obscure the total picture of the problem. Therefore, we apply a body load to D1 with amplitudes $F_{i}=\partial \sigma_{i j} / \partial x_{j}$. The latter is determined from Eqs. (2) and (3), where the displacement field $u_{i}$ is defined by the $\mathrm{RSW}^{5,6}$

$$
u_{i}=\sum_{n}^{3} A^{(n)} u_{0 i}^{(n)} \exp \left[\mathrm{i} q\left(l_{1} x_{1}+l_{2}^{(n)} x_{2}\right)\right]
$$

The above equation is a superposition of three waves with appropriate weighting factors $A^{(n)}$ and amplitudes $u_{0 i}^{(n)}$, where $q=(2 \pi f) / v_{R}$. The considered RSW propagates in the $x_{1} \|[110]$ direction with phase velocity $v_{R}=5087(\mathrm{~m} / \mathrm{s})$ and direction cosine $l_{1}=1$. The values of $l_{2}^{(n)}$ describing the attenuation of RSW amplitude with depth are tabulated together with $A^{(n)}$ and $u_{i 0}^{(n)}$ in the Appendix. Once the strain tensor is determined the values of $F_{i}$ are calculated from the expressions

$$
\begin{gathered}
F_{1}=C_{11}^{\prime} \frac{\partial u_{11}}{\partial x_{1}}+C_{12} \frac{\partial u_{22}}{\partial x_{2}}+2 C_{44} \frac{\partial u_{12}}{\partial x_{2}}, \\
F_{2}=C_{11} \frac{\partial u_{22}}{\partial x_{2}}+C_{12} \frac{\partial u_{11}}{\partial x_{2}}+2 C_{44} \frac{\partial u_{12}}{\partial x_{1}}, \\
F_{3}=0 .
\end{gathered}
$$

Obviously, the above does not apply to the remaining domains, for which $F_{i}=0$. The elastic properties for the domains D1, D2, D3, and D4 are given by the rotated elastic constants $C_{K L}^{\prime}$ and mass density $\rho$. The natural but unwanted reflections from the boundaries due to the finite size of the system are avoided by means of three damping domainsD5, D6, and D7. Similarly, as in the previously described model, we use a position-dependent attenuation resulting from the artificial viscosity (see Eq. (4)). The amplitudes of the incident, reflected, and transmitted RSWs are determined by a spatial fast Fourier transform (FFT) of two line probes with a length of $10 \lambda$ and placed with a distance of $5 \lambda$ before and after the PnC.

\section{RESULTS AND DISCUSSION}

\section{A. Dispersion relation}

In practice, calculations of SAWs dispersion diagrams require a parametric sweep of the wave number $q$ within the IBZ. The value of $q$ defines the periodic boundary conditions of the model and the height of the unit cell. Therefore, to 
avoid a singularity at $q=0$, which results in an infinitely high unit cell, we start calculations from a very small, but nonzero wave number. To sort out SAWs from all FEM solutions, we use a parameter $\xi$, which points the centre of the elastic energy in the $x_{2}$ axis

$$
\xi=\left(1-\frac{\int_{S} E x_{2} \mathrm{~d} S}{H \int_{S} E \mathrm{~d} S}\right),
$$

where the integrals are taken over the unit cell area $S, H$ is the total height of the unit cell, and $E=\frac{1}{2} C_{i j k l} u_{i j} u_{k l}$ is the free elastic energy density. For the considered case, $E$ takes the form ${ }^{3}$

$$
\begin{aligned}
E= & \frac{1}{4}\left(C_{11}\left(u_{11}^{2}+2 u_{22}^{2}\right)+C_{12}\left(u_{11}^{2}+4 u_{11} u_{22}\right)\right. \\
& \left.+2 C_{44}\left(u_{11}^{2}+4 u_{12}^{2}\right)\right) .
\end{aligned}
$$

Generally $\xi$ is a fraction with values from 0 to 1 , where surface-like solutions are those with $\xi$ approaching 0 . In this work, we assume that SAWs are given by $\xi \in(0,0.2)$. Nevertheless, this range depends on the geometrical and material properties applied to the unit cell and has to be defined individually. Nevertheless, $\xi$ does not provide any information that could allow us to differentiate pseudo- and true-SAWs. At this point, we take advantage of the additional damping domain E2 shown in Fig. 1(a). If the unit cell is sufficiently high, then the displacement field of the true-SAW is located near to the free surface of E1 and the displacement in E2 is negligible. Consequently, the additional damping is not required, E2 remains passive, and all the eigenfrequencies are real numbers. This circumstance changes when FEM eigenmodes correspond to pseudo-SAWs. Then, the small radiating component of these waves result in nonzero displacement of E2 and activates the damping properties of this domain. As a consequence, FEM eigenfrequencies are complex numbers with a typically small imaginary part, which decreases together with the magnitude of the leaking component of the displacement. In other words, the surface-like solutions for which $\operatorname{Im}(f)=0$ and $\operatorname{Im}(f) \neq 0$ correspond to true- and pseudo-SAWs, respectively. Figure 2 depicts three exemplary band diagrams of SAWs propagating in PnCs with the same period $a=500 \mathrm{~nm}$ but differing in the size of the stripe. The dotted and dashed lines highlighting the local resonances (LR0, LR1, and LR2) and bulk acoustic waves (LBAW and T1BAW), respectively, are added as a reference. The local resonances are defined by a discrete spectrum of mechanical modes of the isolated stripe that can be tuned by changing its geometrical features. From all the FEM solutions, only those displaying the surface-like parameter $\xi<0.2$ are plotted using two colour scales for true- (red) and pseudo-SAWs (blue). Furthermore, the type of SAWs and features such as penetration depth and leaking can be determined by exploiting FEM displacement fields. The evolution of the total displacement amplitude of the unit cell with depth can be described by its projection on the $x_{2}$ axis given by the formula

$$
\alpha\left(x_{2}\right)=\frac{\int_{S}\left(u_{01}^{2}+u_{02}^{2}\right)^{\frac{1}{2}} \mathrm{~d} x_{1}}{\int_{S} \mathrm{~d} x_{1}} .
$$

In principle, the SAW penetration depth seems to be an arbitrary value, usually taken as few wavelengths. ${ }^{4,6}$ In this work, we define this depth as the distance from the free surface where $\alpha\left(x_{2}\right)$ is $e^{-10}$ smaller than its maximum value.
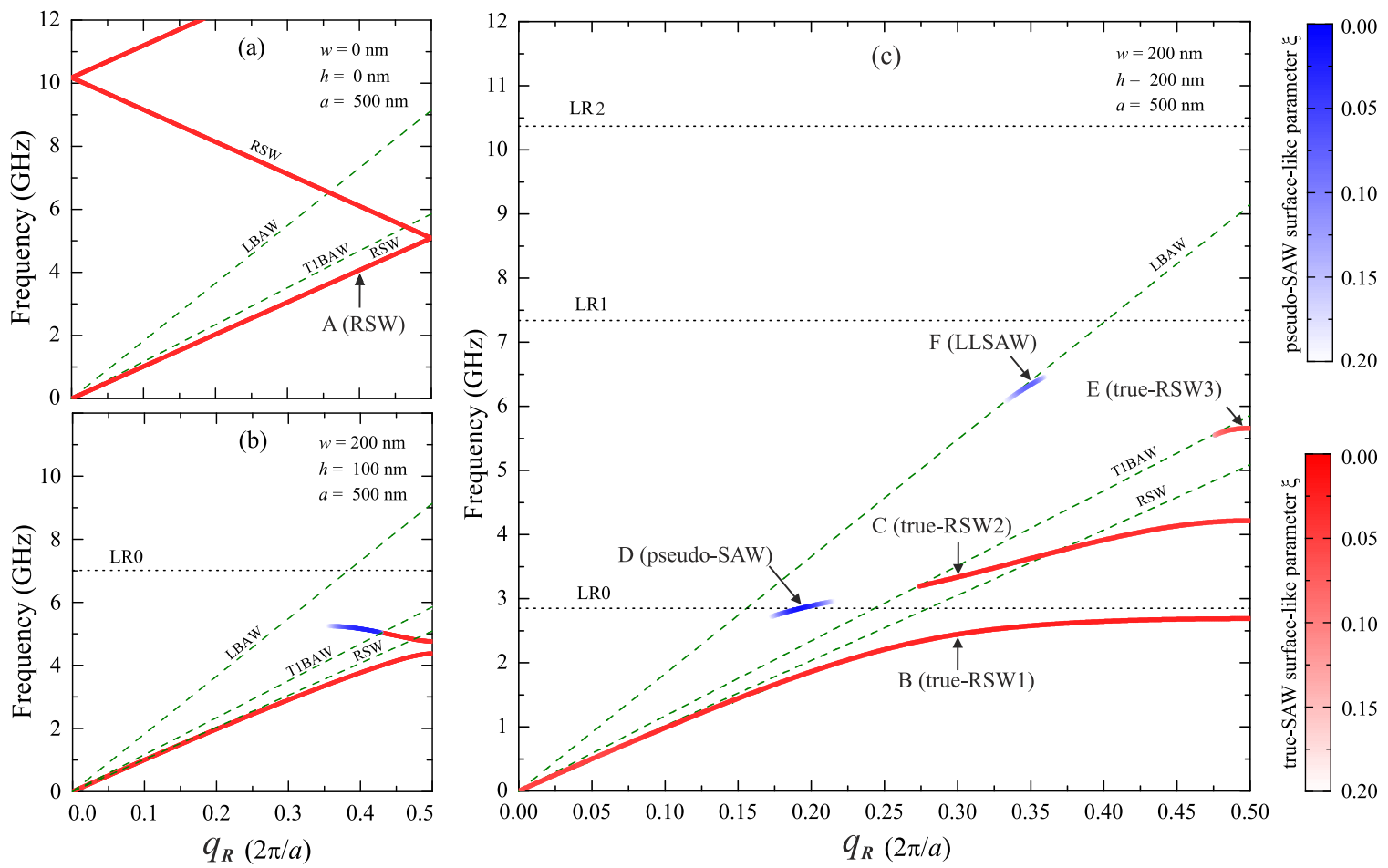

FIG. 2. Exemplary band diagrams of SAWs propagating in PnCs differing in sizes of the stripe (description in the text). 
(a)

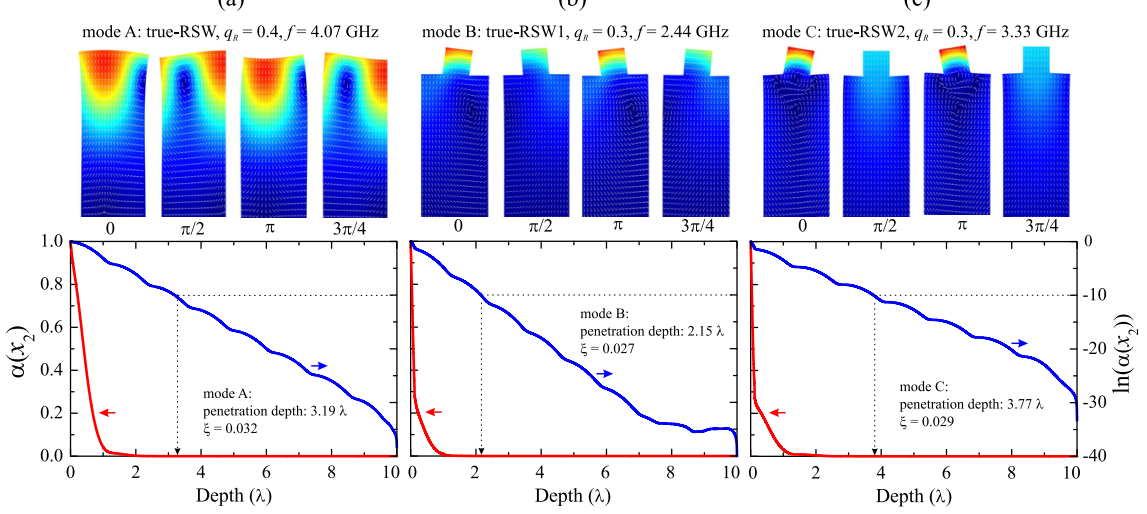

(d)

(e)

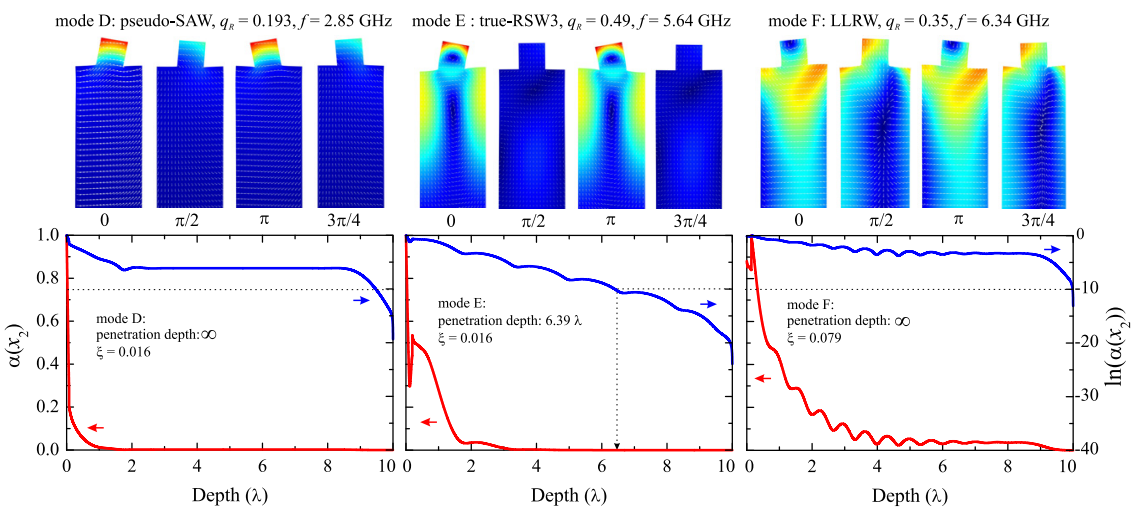

FIG. 3. Displacement fields for different phases and corresponding projected amplitude of the total displacement $\alpha\left(x_{2}\right)$ as a function of depth of modes depicted in Fig. 2.
Figure 2(a) obtained for the unperturbed (001) surface of Si shows nothing more than a dispersion of RSWs propagating in the [110] direction. Naturally, the artificially applied periodicity results in nonphysical zone folding. Nevertheless, in this simple example, the proposed model properly sorts surface-like solutions and selects all of them as true-RSWs even when they are placed above the fast transverse wave threshold (T1BAW). An exemplary RSW at reduced wave number $q_{R}=0.4\left(q_{R}=q a / 2 \pi\right)$ marked as mode A in Fig. 2(a) is depicted in upper Fig. 3(a) in the form of displacement fields at four different phases. Here, as in the next examples of Fig. 3, we take the wave propagation to the right. Automatically, we find the particle motion (arrows) on the free surface as counterclockwise, which is a typical feature of RSWs. Lower part of Fig. 3(a) shows the evolution of the projected total displacement, given by Eq. (12), with depth in linear and logarithmic scales. From this figure and using the above definition, we find the penetration depth to be $3.19 \lambda$.

The second exemplary band structure obtained for a PnC made of stripes with width $w=200 \mathrm{~nm}$ and height $h$ $=100 \mathrm{~nm}$ is depicted in Fig. 2(b). Herein, the periodic perturbation of the surface leads to zone folding of RSWs and Bragg band gap at the zone boundary. All the visible branches demonstrate RSW-like nature, i.e., the free surface particle motion is elliptical and counterclockwise. Both trueRSWs have small $\xi$, and thereby, they are localised very close to the free surface. Furthermore, they are limited by the fast transverse wave threshold. At first glance, the zone folded true-RSW branch seems to transform smoothly into the pseudo-RSW at the crossing point with the T1BAW. However, there is no intermediate state between these two types of waves and the transition is discontinuous. Moreover, the pseudo-RSWs gradually increase the penetration depth and finally vanish while moving from the T1BAW to the LBAW. This behaviour can be attributed to the Brekhovskikh attenuation of SAWs, which is induced by the periodicity and occurs above the transverse wave threshold. ${ }^{15,18,35,41}$ All the frequencies, in the considered range, which lie above the pseudo-RSW branch are forbidden for SAWs. However, contrarily to bulk or plate PnCs, the presence of this wide stop band does not have to result in the full reflection of the incident wave. On the contrary, the reflection is only partial, and the incident wave undergoes partial conversion into BAWs. The latter, considered for finite PnCs in Sec. III B, results in losses of the elastic energy confined to the free surface. The local resonance LR0 appears within the wide band gap above the pseudo-RSWs without any interaction with propagating waves, which could be manifested as SAWs in the dispersion relation. We shall use the next example shown in Fig. 2(c) to analyse in detail the properties of the particular modes, labelled B, C, D, E, and F, as representatives of the corresponding branches. This PnC differs from the previous one only in the height $(h=200 \mathrm{~nm})$ of the stripe. As previously discussed, true-SAWs forming three branches are limited by the transverse wave threshold whereas two other corresponding to pseudo-SAWs appear between the T1BAW and LBAW thresholds. Besides this general similarity, the increased height of the stripe leads to significant changes in the dispersion of SAWs. First of all, 
the eigenfrequencies of the separated stripe, which define the local resonances, are downshifted so one of them, LR0, crosses sound lines of RSW, T1BAW, and LBAW inside the IBZ. In this way, similar to the case of bulk PnCs or metamaterials, it gives rise to anti-crossing of propagating modes accompanied by a reduction in the group velocity and opening of sub-wavelength band gaps. ${ }^{32,42}$ The lowest branch from the non-radiative zone, labelled true-RSW1, spans the whole IBZ; however, it is limited by horizontal and oblique asymptotes given by LR0 and RSW, respectively. A particular mode (mode B in Fig. 3(b)) at $q_{R}=0.3$ shows the typical displacement field of the RSW-like wave: a particle motion on the free surface is counterclockwise for both substrate and stripe. While approaching the zone boundary, the penetration depth of true-RSW1 decreases and the local resonance dominates coupling with RSWs of the substrate. This leads to flattening of the dispersion and reduction in the group velocity. The upper branch, true-RSW2 has a cutoff wave number determined by the fast transverse wave threshold. The displacement fields at $q_{R}=0.3$ (see upper part of Fig. 3(c)) show a counterclockwise particle motion on the surface of the substrate. However, for the surface of the stripe, it is opposite. This, together with the cutoff of the phase velocity given by the T1BAW, makes true-RSW2 similar to Sezawa waves. ${ }^{4,6}$ As follows from Figs. 3(a)-3(c), these waves decay slower with depth than the RSWs of the substrate and the true-RSW1 with the same wave number. Thus, in general, the penetration depth also depends on the wave number.

The sub-wavelength and indirect band gap between true-RSW1 and true-RSW2 certainly applies to true-SAWs, but it does not rule out some nonzero transmission in finite PnCs. The possible transmission may appear via pseudoSAWs formed by the hybridisation of the local resonance LR0 with bulk waves propagating in the substrate, what we shall discuss in Sec. III B. Figure 3(d) shows features of a specific case, a mode $\mathrm{D}$ at $q_{R}=0.193$, where the radiative component of the total projected displacement is only about $e^{-6.13}$ of its maximum value on the free surface. Moreover, the surface-like parameter $\xi$ is about two times smaller than for the RSW of unperturbed $\mathrm{Si}$. In this case, the particle motion of both substrate and stripe is clockwise what makes these waves substantially different from RSWs. The last branch formed in the non-radiative region, true-RSW3, terminates at the fast transverse wave threshold and appears in a very narrow range of wave numbers just next to the zone boundary. As we see in Fig. 3(e) at $q_{R}=0.49$, the penetration depth of the mode $\mathrm{E}$ goes down to $6.39 \lambda$, which is two times deeper than for RSWs. The particle motion of the free surface is counterclockwise as for RSWs; however, the displacement fields of these two waves (compare upper Figs. 3(a) and 3(e)) are completely different. The indirect band gap, which rises between true-RSW2 and true-RSW3 is defined partially at the zone boundary (the lower limit) and on the transverse wave threshold. Similarly, to the previous one, it appears in the sub-wavelength regime, but this time, it applies for both true- and pseudo-SAWs. In the considered range of frequencies, the true-RSW3 at the zone boundary determines the lower limit of a wide stop band for true-
SAWs. However, the leaky branch present at about $6.3 \mathrm{GHz}$ just below the LBAW threshold may result in a nonzero transmission of SAWs through finite PnCs. The dispersion together with displacement fields and evolution of $\alpha\left(x_{2}\right)$ with depth allow us to classify these waves as longitudinal leaky surface acoustic waves (LLSAWs). In principle, longitudinal waves propagating through the free surface are attenuated within a distance of a wavelength. Nevertheless, as was shown, e.g., for $\mathrm{Si}$ and $\mathrm{LiNbO}_{3}$, a periodically corrugated surface can support the propagation of LLSAWs. ${ }^{14,43,44}$

\section{B. Transmission, reflection, and losses}

In this section, we show results of the FEM frequency response study based on the model geometry described in Fig. 1(b) and made out of finite PnCs (100 periods) surrounded by unperturbed silicon. Here, we analyse PnCs with the same spacing and stripe width as in Sec. III A while the height is used as a tuning parameter. As we have assumed, on the left hand side of the PnCs, the model generates RSWs at a given frequency $f$ and without bulk-like components. What is important, RSWs scattered on a surface imperfection can create leaky bulk waves, which if reaching the probe may affect the results. To overcome this issue, we use two line probes placed on two sides of the PnC (see Fig. 1(b)), which determine out-of-plane displacement $u_{2}$ in a line segment of length $10 \lambda$. RSWs propagating in a pristine surface of $\mathrm{Si}$ are non-dispersive; thus, for the frequency $f$, the amplitudes of the incident, reflected, and transmitted RSWs are given as the outputs of the FFT of the corresponding displacement profiles at $1 / \lambda=f / v_{R}$. The transmission $T$, reflection $R$, and surface-to-bulk losses $B$ coefficients are defined as follows:

$$
T=\left(\frac{u_{02}^{T}}{u_{02}^{I}}\right)^{2}, \quad R=\left(\frac{u_{02}^{R}}{u_{02}^{I}}\right)^{2}, \quad B=1-T-R
$$

where $u_{02}^{I}, u_{02}^{T}$, and $u_{02}^{R}$ are amplitudes of the out-of-plane displacement of incident, transmitted, and reflected RSWs, respectively. The spectra of $T, R$, and $B$ are obtained by sweeping the frequency $f$, and thereby $q$ in Eq. (6), in a range of $0.01-12 \mathrm{GHz}$. Before going into a more general picture, let us consider a specific case of $\mathrm{PnC}$, the dispersion relation of which we have already analysed in detail. Figure 4(a) depicts the dispersion relation, the same shown in Fig. 3(c). However, here, we marked the band gaps for true- and pseudo-SAWs, and we shall use it as complementary data for the frequency response study. In addition to the finite PnCs, we examine a reference system where the periodic array is substituted by a single building element, a stripe. As follows from Figs. 4(b)-4(d), all three coefficients $T, R$, and $B$, respectively, are frequency dependent. While increasing the frequency the wavelength and penetration depth of RSWs become comparable to the stripe dimensions. This is generally accompanied by an increase of $B$ and a reduction of $T$. In our case, this trend is modified, at different levels, by the presence of three local resonances LR0 $(2.85 \mathrm{GHz}), \mathrm{LR} 1$ $(7.34 \mathrm{GHz})$, and LR2 $(10.37 \mathrm{GHz})$. The effect of the fundamental mechanical eigenmode of the stripe is stronger, but 


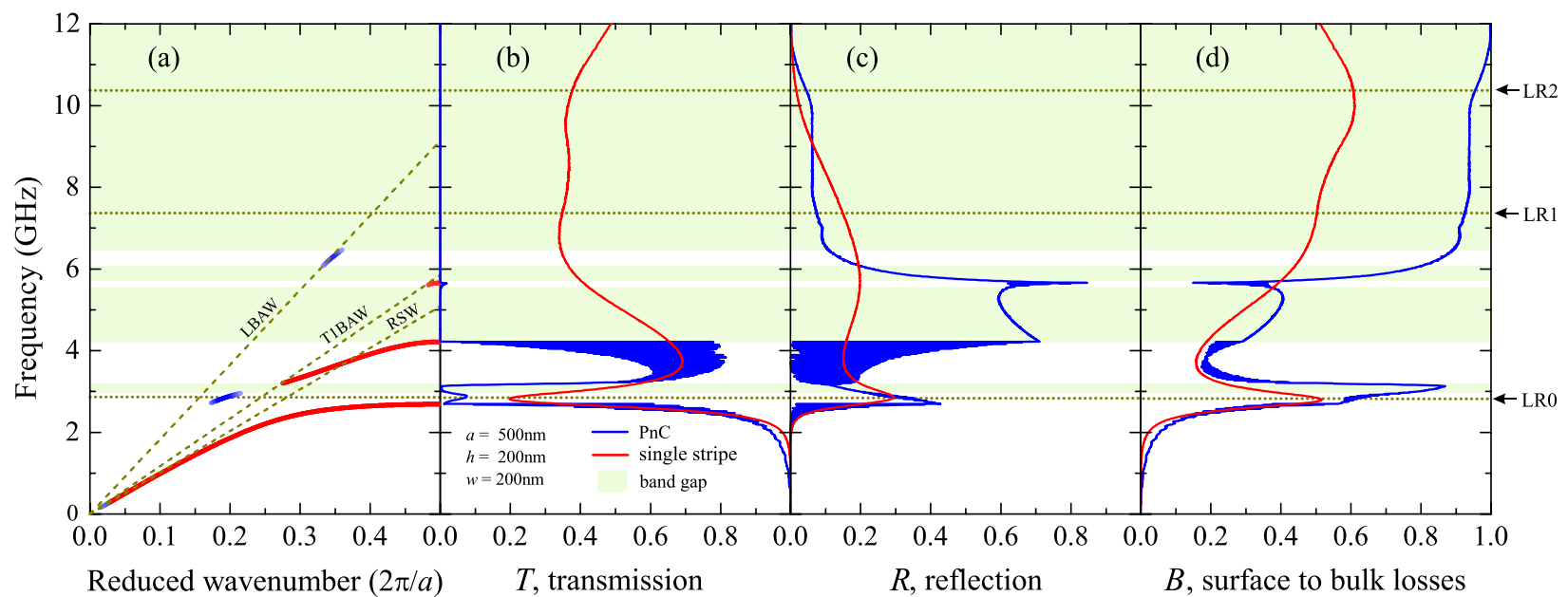

FIG. 4. (a) Dispersion relation of SAWs taken from Fig. 2(c) and corresponding coefficients of (b) transmission $T$, (c) reflection $R$, and (d) surface-to-bulk losses $B$ plotted for a $\mathrm{PnC}$ and single stripe as a reference.

all local resonances give rise to deeps of $T$ and peaks $R$ and $B$. We now turn on to examine the PnCs with respect to the results for the single stripe. As we see in Fig. 4, the predicted band gaps in the dispersion relation perfectly match bandwidths of zero transmission. Up to approximately $2.63 \mathrm{GHz}, T$ is carried via true-RSW1 and resembles the behaviour of the single stripe. As we expected, the propagation of SAWs is not completely cancelled inside the

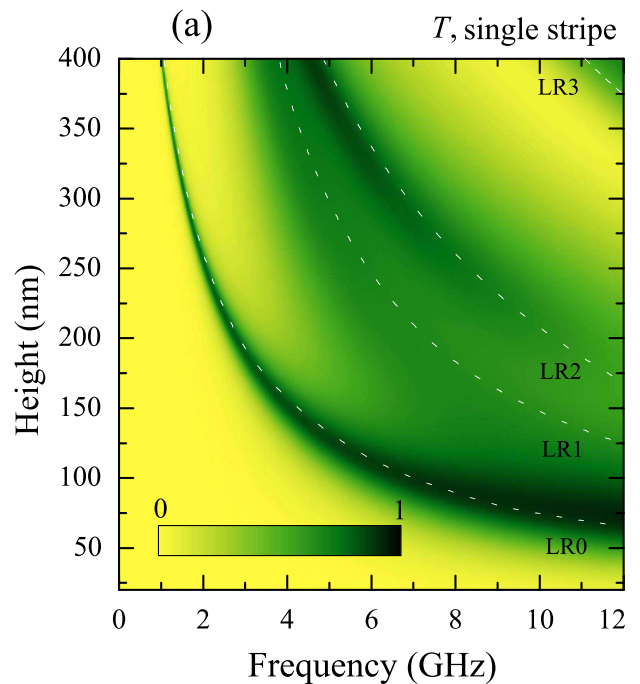

(d)

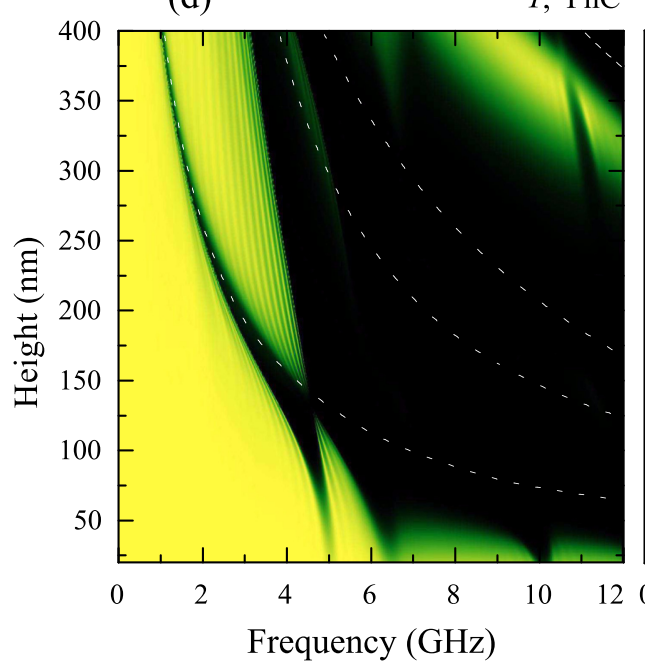

(b)

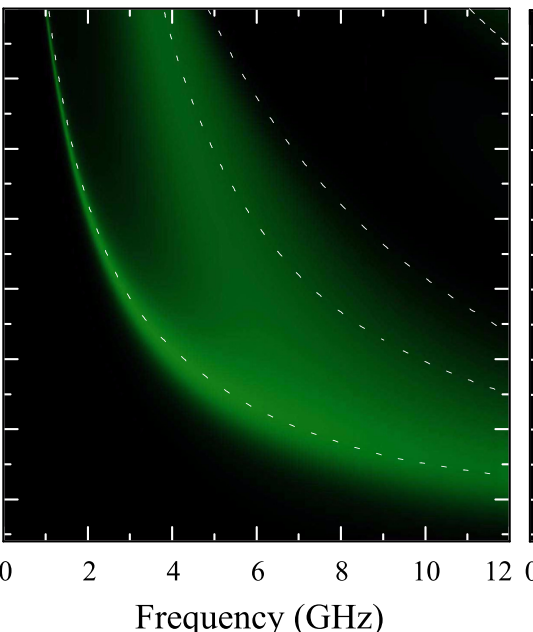

(e)

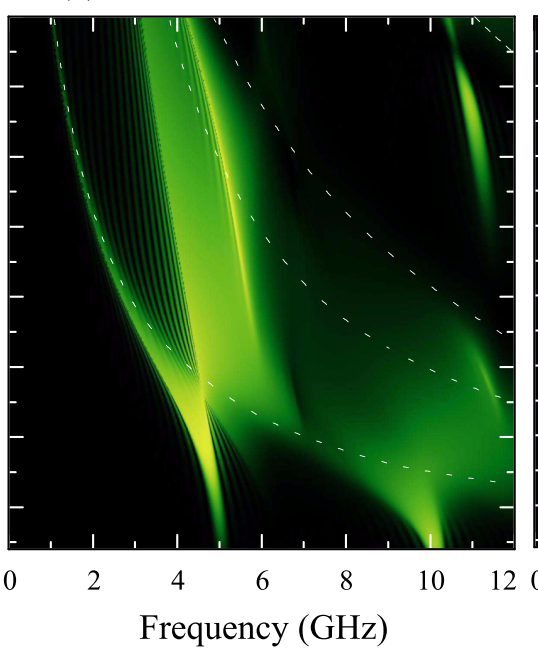

(c)

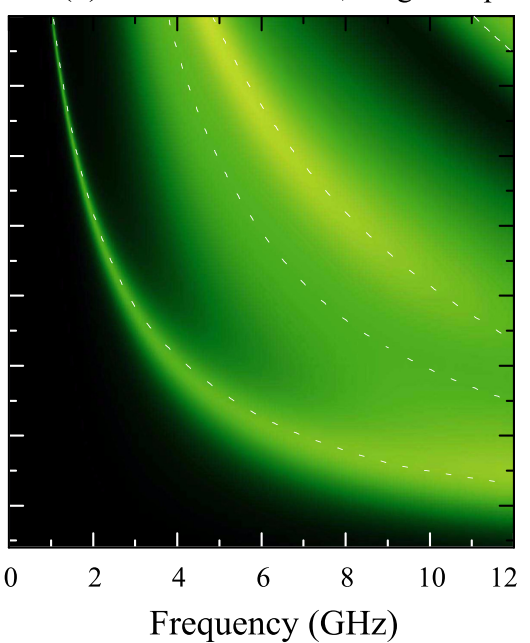

(f)

$B, \mathrm{PnC}$

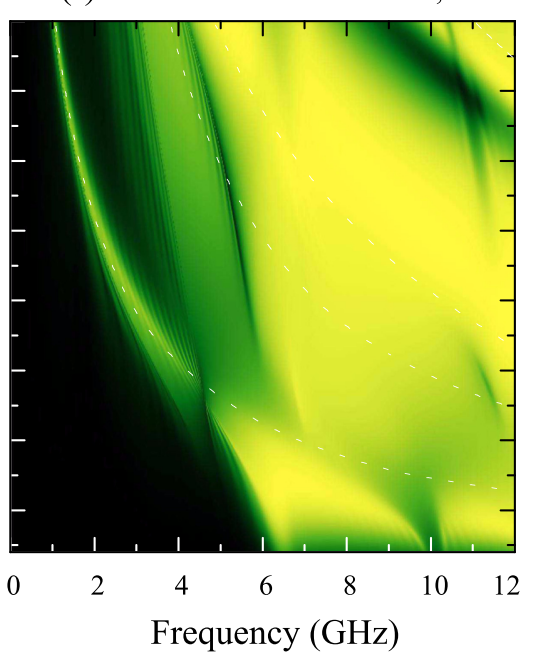

FIG. 5. Coefficients of transmission $(T)$, reflection $(R)$, and surface-to-bulk losses $(B)$ as a function of frequency and height of the stripe calculated for the model geometry of Fig. 1(b) made out of (a)-(c) a single stripe and (d)-(f) PnCs with 20 periods. 
non-radiative band gap between true-RSW1 and trueRSW2. Interestingly, the deep of $T$ at $2.85 \mathrm{GHz}$ found for the single stripe turns into a peak in the case of the PnC. Although $T$ at that point is relatively smaller for the $\mathrm{PnC}$, RSWs are clearly transmitted through the PnC via pseudoSAWs. Going to higher frequencies, SAWs are effectively stopped within a narrow range defined between branches of pseudo-SAWs and true-RSW2. The transmission via trueRSW2 remains high but lower than 0.8 due to partial reflection and conversion to BAWs at the interfaces of the PnC. The pass band of true-RSW3 shows relatively small $T$ while that of LLSAWs is practically not visible. From the viewpoint of potential applications of PnCs as SAWs reflectors, the reflection coefficient $R$ has to be maximised, what is not trivial when surface-to-bulk losses become an important issue. ${ }^{40,45}$ As we see in Fig. 4(c), the reflectivity related to LR0 is about 0.4 , and this within the second band gap varies between 0.6 and 0.7. However, the maximum $R=0.85$ appears as a narrow peak at $5.66 \mathrm{GHz}$ within the third band-gap just above true-RSW3. This situation is accompanied by very curious behaviour of surface-to-bulk losses. At this point, $B$ for PnCs has a deep and surprisingly is about 2.6 times lower than that for the single stripe. This finding suggests that the interface between the flat surface and $\mathrm{PnCs}$ is not defined by the first single stripe. Consequently, we can optimise $R$ within a given range of frequencies by an appropriate design of the PnCs. As a summary of this section, we show an exemplary optimisation study, which in practice is an extension of the case shown in Fig. 4. All three coefficients $T, R$, and $B$ are calculated as a function of frequency and height of the stripe and plotted in a colour scale. Figures 5(a)-5(c) show results for the reference system build of a single stripe with fixed width and varied height in a range of $20-400 \mathrm{~nm}$. Here again, the presence of the local resonances clearly manifested in the 2D maps of $T, R$, and $B$. In principle, they lead to reduction of $T$ and simultaneous increase of $R$ and $B$. Similar calculations showed in Figs. 5(d) $-5(\mathrm{f})$ were performed for PnCs made of 20 stripes with fixed spacing and width and height varied as in the example above. From the comparison with the previous case, we can conclude that the periodic array decreases the transmission in a favour of mainly the radiation to bulk. These adverse losses under certain frequencies and sizes can be minimised and accompanied by very high reflectivity. Herein, the overall picture points to LR0 as only one from all the mechanical eigenmodes of the stripe, which substantially affects $T, R$, and $B$.

\section{CONCLUSIONS}

Making use of FEM, we studied SAWs dispersion and propagation through PnCs made of an array of rectangular stripes on the (001) surface of $\mathrm{Si}$. A new approach within a framework of the eigenfrequency study allowed us to overcome the limitation of FEM in the form of a finite height unit cell. By applying an additional viscoelastic domain we sorted true- and pseudo-SAWs without resorting to the sound cone criterion. In this way, we avoided imposing a fixed displacement boundary condition, which affects the radiative component and, consequently, the dispersion of pseudoSAWs. We showed dispersion features such as zone folding, band gaps originating from Bragg reflections and local resonances, the latter splitting RSWs into Rayleigh- and Sezawa-like waves, and the presence of LLSAWs. We further performed a comparative and complementary study of transmission, reflection, and surface-to-bulk losses in finite PnCs. The novelty of the developed FEM frequency response model is based on the generation of pure RSWs and its analysis by the spatial FFT. The obtained results showed that the local resonances, driven by geometrical features of the stripe, lead to opening of sub-wavelength band gaps. Moreover, in the same bandwidth, they hybridise with propagating bulk waves and give rise to pseudo-SAWs with a very small leaky component. This leads directly to nonzero transmission of RSWs through a finite PnC. We uncovered an interesting phenomenon which is manifested by decreasing surface-to-bulk losses in PnCs with respect to a single stripe. This feature appears within a band gap, just above trueRSW3, and is accompanied by a sharp increase in the reflectivity. The presented approach shows a novel insight on the propagation of true- and pseudo-SAWs in PnCs, which can be useful for the design and optimisation of devices employing SAWs.

\section{ACKNOWLEDGMENTS}

The authors acknowledge the financial support from the FP7 project MERGING (Grant No. 309150); the Spanish MICINN projects nanoTHERM (Grant No. CSD2010-0044) and TAPHOR (MAT2012-31392); and the program Severo Ochoa (Grant SEV-2013-0295).

\section{APPENDIX: COEFFICIENTS $A^{(n)}, u_{0 i}^{(n)}$, AND $l_{2}^{(n)}$}

The coefficients $A^{(n)}, u_{0 i}^{(n)}$, and $l_{2}^{(n)}$, which appear in Eq. (6), can be obtained by, e.g., the partial waves approach. ${ }^{5,6}$ Using this formalism, we find velocity of RSWs as $v_{R}=5087 \mathrm{~m} / \mathrm{s}$ and corresponding coefficients gathered in Table II.

TABLE II. Coefficients $u_{0 i}^{(n)}, l_{2}^{(n)}$, and $A^{(n)}$.

\begin{tabular}{lcc}
\hline \hline 1 & $l_{2}^{(1)}$ & $-0.4041828618678606-0.5314332666932391 \mathrm{i}$ \\
2 & $l_{2}^{(2)}$ & $0.4041828618678606-0.5314332666932391 \mathrm{i}$ \\
3 & $l_{2}^{(3)}$ & $0.3433811825660294+0 \mathrm{i}$ \\
4 & $u_{01}^{(1)}$ & $0.473292646309311+0.361528977733201 \mathrm{i}$ \\
5 & $u_{01}^{(2)}$ & $-0.473292646309311+0.361528977733201 \mathrm{i}$ \\
6 & $u_{01}^{(3)}$ & $0+0 \mathrm{i}$ \\
7 & $u_{02}^{(1)}$ & $0.803299987058830+0 \mathrm{i}$ \\
8 & $u_{02}^{(2)}$ & $0+0 \mathrm{i}$ \\
9 & $u_{02}^{(3)}$ & $0+0 \mathrm{i}$ \\
10 & $u_{03}^{(1)}$ & $-1+0 \mathrm{i}$ \\
11 & $u_{03}^{(2)}$ & $A(1+0 \mathrm{i})$ \\
12 & $u_{03}^{(3)}$ & $A(0+0 \mathrm{i})$ \\
14 & $A^{(1)}$ & $A(-0.607025491288873+0.794682359767411 \mathrm{i})$ \\
14 & $A^{(2)}$ & \\
15 & $A^{(3)}$ & \\
\hline \hline
\end{tabular}


${ }^{1}$ L. Rayleigh, Proc. London Math. Soc. 17, 4 (1885).

${ }^{2}$ I. A. Viktorov, Rayleigh and Lamb Waves: Physical Theory and Applications (Plenum Press, New York, 1967).

${ }^{3}$ L. D. Landau and E. M. Lifshitz, Theory of Elasticity (Pergamon Press, New York, USA, 1959).

${ }^{4}$ B. A. Auld, Acoustic Fields and Waves in Solids (Krieger Publishing Company, USA, 1990), Vol. 2.

${ }^{5}$ G. W. Farnell, in Properties of Elastic Surface Waves, Physical Acoustics Vol. 6, edited by W. P. Mason and R. N. Thurston (Academic Press, New York, 1970), pp. 109-166.

${ }^{6}$ G. W. Farnell and E. L. Adler, Elastic Wave Propagation in Thin Layers, Physical Acoustics Vol. 9 (Academic Press, New York, 1972), pp. 35-127.

${ }^{7}$ V. R. Velasco and F. Garcia-Moliner, J. Phys. C: Solid State Phys. 13, 2237 (1980).

${ }^{8}$ R. Hays and C. Hartmann, Proc. IEEE 64, 652 (1976).

${ }^{9}$ H. S. Tuan and J. P. Parekh, Appl. Phys. Lett. 30, 220 (1977).

${ }^{10}$ D. A. Simons, J. Acoust. Soc. Am. 63, 1292 (1978).

${ }^{11}$ S. R. Seshadri, J. Acoust. Soc. Am. 65, 687 (1979).

${ }^{12}$ N. E. Glass, R. Loudon, and A. A. Maradudin, Phys. Rev. B 24, 6843 (1981).

${ }^{13}$ J. R. Dutcher, S. Lee, B. Hillebrands, G. J. McLaughlin, B. G. Nickel, and G. I. Stegeman, Phys. Rev. Lett. 68, 2464 (1992).

${ }^{14}$ S. Lee, L. Giovannini, J. R. Dutcher, F. Nizzoli, G. I. Stegeman, A. M. Marvin, Z. Wang, J. D. Ross, A. Amoddeo, and L. S. Caputi, Phys. Rev. B 49, 2273 (1994).

${ }^{15}$ A. A. Maradudin and W. Zierau, Geophys. J. Int. 118, 325 (1994).

${ }^{16}$ Y. Tanaka and S.-I. Tamura, Phys. Rev. B 58, 7958 (1998).

${ }^{17}$ T. T. Wu, Z. G. Huang, and S. Lin, Phys. Rev. B 69, 094301 (2004).

${ }^{18}$ A. A. Maznev, Phys. Rev. B 78, 155323 (2008).

${ }^{19}$ L. Dhar and J. A. Rogers, Appl. Phys. Lett. 77, 1402 (2000).

${ }^{20}$ B. Graczykowski, M. Sledzinska, N. Kehagias, F. Alzina, J. S. Reparaz, and C. M. Sotomayor Torres, Appl. Phys. Lett. 104, 123108 (2014).

${ }^{21}$ S. Brûlé, E. H. Javelaud, S. Enoch, and S. Guenneau, Phys. Rev. Lett. 112, 133901 (2014).

${ }^{22}$ B. L. Davis and M. I. Hussein, Phys. Rev. Lett. 112, 055505 (2014).

${ }^{23}$ J. Gomis-Bresco, D. Navarro-Urrios, M. Oudich, S. El-Jallal, A. Griol, D. Puerto, E. Chavez, Y. Pennec, B. Djafari-Rouhani, F. Alzina, A. Martínez, and C. M. S. Torres, Nat. Commun. 5, 4452 (2014).

${ }^{24}$ M. Maldovan, Nature 503, 209 (2013).
${ }^{25}$ B. Graczykowski, B. Mroz, S. Mielcarek, T. Breczewski, M. L. Nó, and J. S. Juan, J. Phys. D: Appl. Phys. 44, 455307 (2011).

${ }^{26}$ J. D. Comins, in Handbook of Elastic Properties of Solids, Liquids and Gases, edited by A. Every and W. Sachse (Academic Press, San Diego, 2001), Vol. 1, pp. 349-378.

${ }^{27}$ X. Zhang, J. D. Comins, A. G. Every, P. R. Stoddart, W. Pang, and T. E. Derry, Phys. Rev. B 58, 13677 (1998).

${ }^{28}$ J. Sandercock, in Light Scattering in Solids III, Topics in Applied Physics Vol. 51, edited by M. Cardona and G. Gntherodt (Springer, Berlin, Heidelberg, 1982), pp. 173-206.

${ }^{29}$ S. Benchabane, A. Khelif, J.-Y. Rauch, L. Robert, and V. Laude, Phys. Rev. E 73, 065601 (2006).

${ }^{30}$ T.-T. Wu, Z.-C. Hsu, and Z.-G. Huang, Phys. Rev. B 71, 064303 (2005).

${ }^{31}$ V. Laude, M. Wilm, S. Benchabane, and A. Khelif, Phys. Rev. E 71, 036607 (2005).

${ }^{32}$ A. Khelif, Y. Achaoui, S. Benchabane, V. Laude, and B. Aoubiza, Phys. Rev. B 81, 214303 (2010).

${ }^{33}$ Y. Achaoui, A. Khelif, S. Benchabane, L. Robert, and V. Laude, Phys. Rev. B 83, 104201 (2011).

${ }^{34}$ D. Yudistira, Y. Pennec, B. Djafari Rouhani, S. Dupont, and V. Laude, Appl. Phys. Lett. 100, 061912 (2012).

${ }^{35}$ A. A. Maznev and A. G. Every, J. Appl. Phys. 106, 113531 (2009).

${ }^{36}$ S. Mielcarek, A. Trzaskowska, B. Graczykowski, and J. Sarkar, Phys, Status Solidi RRL 6, 175 (2012).

${ }^{37}$ M. Badreddine Assouar and M. Oudich, Appl. Phys. Lett. 99, 123505 (2011).

${ }^{38}$ R. E. Newnham, Properties of Materials: Anisotropy, Symmetry, Structure (Oxford University Press, Oxford, UK, 2005).

${ }^{39}$ B. Hosten, L. Moreau, and M. Castaings, J. Acoust. Soc. Am. 121, 3409 (2007).

${ }^{40}$ B. Graczykowski, J. Appl. Phys. 112, 103520 (2012).

${ }^{41}$ L. M. Brekhovskikh, Sov. Phys. Acoust. 5, 288 (1960).

${ }^{42}$ T. Still, W. Cheng, M. Retsch, R. Sainidou, J. Wang, U. Jonas, N. Stefanou, and G. Fytas, Phys. Rev. Lett. 100, 194301 (2008).

${ }^{43}$ T. Makkonen, V. P. Plessky, W. Steichen, and M. M. Salomaa, Appl. Phys. Lett. 82, 3351 (2003).

${ }^{44}$ O. Holmgren, J. V. Knuuttila, T. Makkonen, K. Kokkonen, V. P. Plessky, W. Steichen, M. Solal, and M. M. Salomaa, Appl. Phys. Lett. 86, 024101 (2005).

${ }^{45}$ Y. Xu and P. Peng, J. Appl. Phys. 117, 035103 (2015). 